\title{
Factores adversos al fomento de hábitos de estudio y aprendizaje en educación superior: Un estudio de caso
}

Adverse factors hindering the promotion of study and learning habits in higher education. A case study

\section{Volumen 18, Número 3 \\ Setiembre-Diciembre}

pp. 1-30

Este número se publica el 1 de setiembre de 2018

DOI: https://doi.org/10.15517/aie.v18i3.34122

Juan Bautista Climént Bonilla

Revista indizada en REDALYC, $\underline{\text { SCIELO }}$

Revista distribuida en las bases de datos:

LATINDEX, DOAJ, REDIB, IRESIE, CLASE, DIALNET, SHERPA/ROMEO, QUALIS-CAPES, MIAR

Revista registrada en los directorios:

ULRICH'S, REDIE, RINACE, OEI, MAESTROTECA, PREAL, CLACSO 


\title{
Factores adversos al fomento de hábitos de estudio y aprendizaje en educación superior: Un estudio de caso
}

Adverse factors hindering the promotion of study and learning habits in higher education. A case study

\section{Juan Bautista Climént Bonilla}

Resumen: La formación de competencias académicas y profesionales requiere de la sistematización y desarrollo compaginado entre habilidades -físicas y cognitivas - y hábitos y rutinas de estudio y aprendizaje apropiados. La investigación, sobre la que se sustenta este artículo, consiste en un estudio de caso cuyo problema central es la falta de buenos hábitos y rutinas de estudio y aprendizaje para el adecuado aprovechamiento de un grupo de 31 estudiantes durante un módulo (curso) de la carrera de Medicina Veterinaria y Zootecnia, que se imparte en la Universidad Autónoma Metropolitana, Unidad Xochimilco (UAM-Xochimilco), en la Ciudad de México. El propósito principal de la investigación es la identificación y análisis de los factores adversos al fomento de buenos hábitos de estudio y aprendizaje en el contexto del caso. Las particularidades de la situación observada se analizan desde distintos ángulos de interés, en el marco de una investigación cualitativa. Destaca la identificación de seis factores adversos: tres en el carácter de hábitos y rutinas que compiten con los hábitos de estudio y aprendizaje deseados, y tres más, producto de las circunstancias, contingencias e imprevistos que dificultan u obstaculizan la formación del alumnado, especialmente en aspectos concernientes al aprendizaje procedimental de habilidades físicas y cognitivas. Los hallazgos reportados enfatizan la importancia de observar los desafíos de la educación superior desde una amplia perspectiva de contextos.

Palabras clave: enseñanza superior, hábitos de estudio, adquisición de conocimientos, desarrollo de las habilidades

\begin{abstract}
Achievement of academic and professional competences requires the systematization and developmental balance among physical and cognitive skills and suitable habits and routines of study and learning. The research, on which this article is based, consists of a case study whose main problem is the lack of good habits and routines of study and learning for the academic progress of a group of 31 students throughout a module (course) of the Veterinary Medicine Career, which is taught at the Universidad Autónoma Metropolitana, campus Xochimilco (UAM-Xochimilco), in Mexico City. The main purpose of this research is the identification and analysis of adverse factors hindering the promotion of good study and learning habits in the context of the case study. The characteristics of the observed situation are analyzed from different approaches of interest within the framework of qualitative research. The identification of six adverse factors is highlighted: three of them as habits and routines that compete with the desired study and learning habits; and three more, as a result of the circumstances, contingencies, and unforeseen difficulties that complicate or hinder students' academic achievement, especially in aspects concerning the procedural learning of physical and cognitive skills. The reported findings emphasize the importance of observing higher education challenges from a broad perspective of contexts.
\end{abstract}

Key words: higher education, study habits, knowledge acquisition, skills development

\footnotetext{
1 Profesor e investigador de la Universidad Autónoma Metropolitana, Unidad Xochimilco, en la ciudad de México. Doctor en Educación, por la Universidad de Cornell.
}

Dirección electrónica: jcliment@correo.xoc.uam.mx

Artículo recibido: 8 de noviembre, 2017

Enviado a corrección: 19 de febrero, 2018

Aprobado: 25 de junio, 2018 


\section{Introducción}

En el ámbito de la educación superior, la formación de hábitos de estudio y aprendizaje implica el acondicionamiento progresivo de las facultades físicas y mentales del estudiantado, con el fin de conseguir, acompasadamente, un cúmulo de conocimientos y habilidades académicas y profesionales. Lally, van Jaarsveld, Potts y Wardle (2010) hacen ver que la investigación sistemática sobre conductas repetidas en situaciones consistentes se ha abocado, primero, a los hábitos simples — como los relacionados con prácticas de alimentación, ejercicio y salud - más que a los complejos —como los relativos a actividades regulares y efectivas de estudio y aprendizaje-; segundo, a la evaluación de la frecuencia de conductas pasadas, más que al proceso de formación de estas; y tercero, al desarrollo de conductas habituales fuera del "mundo real", más que en el contexto de situaciones reales. A todo esto, las investigaciones han privilegiado el estudio de la formación de hábitos como un proceso bajo control individual, al margen de condiciones externas, que son parte de la realidad —o las realidades - en que viven y se forman las personas. De hecho, la realidad de las instituciones de educación superior está sujeta a la interacción permanente y multilateral de las actividades académicas con el medio exterior, de modo que todas las universidades del mundo están expuestas, en mayor o menor medida, a las vicisitudes políticas, económicas, sociales y culturales del exterior. Las universidades e instituciones de educación superior mexicanas no son la excepción, día a día deben afrontar las presiones y cambios provenientes del medio que les rodea, en esferas que van desde lo global hasta lo local, y cuyos efectos - algunos favorables y otros adversos- permean en sus funciones sustantivas, en ocasiones, de manera crítica, llegando a repercutir sobre las actividades de enseñanza-aprendizaje a nivel de aula (Del Valle, 2016).

La elaboración del presente artículo se basa en un estudio de caso que tiene por propósito principal identificar y analizar una serie de condiciones con efectos negativos sobre los hábitos de estudio y aprendizaje de un grupo de estudiantes del módulo "Sistemas de Producción Animal", de la carrera de Medicina Veterinaria y Zootecnia, de la Universidad Autónoma Metropolitana, Unidad Xochimilco (UAM-Xochimilco), en la Ciudad de México. EI módulo fue impartido por el autor del artículo el segundo trimestre (mayo a julio) de 2016.

Sobre la base de que el salón de clases es la "unidad funcional" de la docencia principal función sustantiva de las instituciones de educación superior-, la presente investigación parte de dos supuestos interrelacionados:

- Las diferencias entre instituciones, planes de estudio, asignaturas, grupos de estudiantes, estudiantes y docentes, son tan importantes o más que sus similitudes. 
- El conocimiento de las particularidades de un estudio de caso, relativo a los factores que afectan los hábitos de estudio y aprendizaje, aporta a la comprensión de los problemas asociados con la formación del alumnado, en busca de posibles soluciones, adecuaciones y mejoras.

En síntesis, el estudio plantea, como problema central del caso, la falta de buenos hábitos y rutinas de estudio y aprendizaje para el adecuado aprovechamiento de la población estudiantil en el ámbito del módulo de enseñanza-aprendizaje abordado. De dicho problema se desprende el propósito principal de la investigación realizada, la identificación y análisis de los factores adversos al fomento de buenos hábitos de estudio y aprendizaje en el contexto del caso, así como las dos grandes interrogantes a tratar: ¿cuáles son esos factores adversos? y ¿qué aspectos del desempeño académico del estudiantado (habilidades, hábitos y rutinas de estudio y aprendizaje) es pertinente destacar, ante los efectos negativos de tales factores?

\section{Marco contextual}

Las condiciones desfavorables para la formación de hábitos de estudio y aprendizaje, como factores causales, predisponentes o de riesgo del problema central planteado, se analizan en el marco de la interrelación de cuatro áreas de influencia que constituyen los contextos del caso: a) el global, propio de la sociedad del conocimiento y la era de la información, y consustancial al desarrollo de las nuevas tecnologías de la información y la comunicación (TIC); b) el poblacional y urbano, de la Ciudad de México; c) el institucional, de la Universidad Autónoma Metropolitana, Unidad Xochimilco, en la Ciudad de México; y d) el docente, donde se desarrollan las actividades de enseñanza-aprendizaje de un módulo (curso) de la carrera de Medicina Veterinaria y Zootecnia, impartido a un grupo de estudiantes de la misma institución.

En el marco de dicha interrelación, el influjo de condiciones adversas pasa de las esferas externas al medio académico - los dos primeros contextos-, a las esferas internas -los dos últimos contextos-, propias del medio universitario. Concomitantemente, el curso de los eventos que atañen al problema del caso también discurre de las esferas externas a las internas. Por lo que toca al contexto global, el estudiantado involucrado en este estudio de caso forma parte de la llamada sociedad del conocimiento: la sociedad global, cuyos miembros, de manera abierta y sin distinción, tienen la posibilidad de intercomunicarse y acceder a prácticamente todo tipo de información y conocimientos, sin límites de distancia y 
en tiempo simultáneo, mediante medios y mecanismos electrónicos, como computadoras, internet, multimedia, teléfonos "inteligentes" y el sistema de telecomunicaciones que soporta la operación de las llamadas TIC (Climént, 2011, p. 354). El uso de las TIC puede servir a los jóvenes para propósitos formativos (educación, capacitación, cultura, aprendizaje, comunicación), así como también para ocuparlos en actividades no formativas, al punto que el uso excesivo e inadecuado de dispositivos electrónicos puede mermar el potencial de procesos de interiorización y aprendizaje profundo, que están por arriba de la información básica y las habilidades rutinarias.

Es necesario tener presente, en lo relativo al contexto poblacional y urbano, que la Zona Metropolitana del Valle de México (ZMVM) comprende los 16 municipios de la capital del país y 60 municipios conurbados, área geográfica equivalente al $0.4 \%$ del territorio nacional. De acuerdo con datos censales (INEGI, 2015), en el curso de las primeras siete décadas del siglo pasado (1900 a 1970), la población de la Ciudad de México (CDMX) creció cerca de 10 veces, pasando de .7 a 6.9 millones de habitantes. A los 6.9 millones de habitantes de principios de la década de 1970, se sumaron 2 millones más en el transcurso de los siguientes 45 años, con lo que la población alcanzó, en 2015, 8.9 millones de personas: un 29\% más que en 1970. Según datos del censo de 2010 (INEGI, 2011; citado por INECC, 2011), la población de la ZMVM era de alrededor de 29 millones de habitantes, cifra que representaba más de la cuarta parte (25.8\%) de toda la población del país (112.3 millones de habitantes), de ese mismo año (INEGI, 2010).

Como consecuencia del crecimiento poblacional y urbano de la ZMVM, se ha incrementado la flota vehicular, el tráfico, las distancias de desplazamiento y, por consiguiente, los tiempos de traslado. Expertos en materia de transporte estiman que el parque vehicular de la ZMVM rebasaba los 5 millones en 2008, y que hoy en día, el excesivo uso y número de automóviles particulares pueden llevar el nivel del aforo vehicular y de los tiempos de traslado en la ZMVM al colapso vial de la megalópolis (Zamudio y Alvarado, 2014, pp. 5, 10).

Ante el pronunciado aumento de la población de la capital del país y consecuentemente, de la demanda de servicios de educación superior en la metrópoli —que atañe al contexto institucional del estudio que nos ocupa-, a instancias de la Asociación Nacional de Universidades e Institutos de Educación Superior (ANUIES), bajo el gobierno del Presidente Luis Echeverría Álvarez, se creó la Universidad Autónoma Metropolitana (UAM) -a partir de la Ley Orgánica de la Universidad Autónoma Metropolitana, publicada en el 
Diario Oficial de la Federación el 17 de diciembre de 1973-,"como organismo descentralizado del Estado, con personalidad jurídica y patrimonio propio" (p. 1).

La UAM nace con tres unidades físicas o planteles: la Unidad Azcapotzalco, la Unidad Iztapalapa y la Unidad Xochimilco, ubicadas estratégicamente en demarcaciones territoriales de la ciudad —llamada Distrito Federal, desde su creación con la constitución de 1824, hasta el 29 de enero de 2016, en que por Decreto en materia de reforma política de la capital del país pasó a denominarse Ciudad de México- para atender las crecientes demandas de educación superior en la metrópoli, especialmente, en zonas de su periferia. Hoy en día, la UAM es la segunda universidad pública más importante de México y opera con cinco unidades, que funcionan de manera coordinada en cuanto a asuntos comunes, pero independiente en temas internos.

Por último, en relación con el contexto docente, donde se realizó el estudio de caso aquí presentado, cabe destacar los siguientes eventos y circunstancias: La UAM-Xochimilco inicia clases el 11 de noviembre de 1974 con un modelo de enseñanza-aprendizaje, distinto al esquema tradicional de asignaturas, basado en módulos que tienen por objeto de estudio e investigación problemas reales, vinculados a situaciones -sociales, culturales, tecnológicas, económicas, medioambientales - concretas, bajo un enfoque integral e interdisciplinario.

Desde sus inicios hasta abril de 2016, la carrera de Medicina Veterinaria y Zootecnia MVZ) se ofreció en la UAM-Xochimilco, con un plan de estudios a cuatro años y 12 módulos, tres por año. A principios de mayo de 2016, con el segundo trimestre académico del calendario escolar 2015-2016, entra en operación un nuevo plan de estudios para la carrera de MVZ en la UAM-Xochimilco. En contraste con el plan anterior (noviembre de 1974 a abril de 2016), el nuevo plan enfatiza la formación veterinaria por encima de la zootécnica y se imparte en cinco años, en lugar de cuatro, y 15 módulos, en vez de 12. Asimismo, en el marco de los procesos globalizadores de nuestro tiempo, entre sus lineamientos destacan: el enfoque de competencias (UAM-Xochimilco, 2013, p. 1), la certificación del programa de educación veterinaria y la apertura internacional a la formación (educación, capacitación y experiencia) de los egresados (Taylor, 2003, pp. 6-7).

Dentro del nuevo plan de estudios, la última etapa del plan anterior, denominada "Crianza Racional", compuesta por tres módulos que se impartían durante el cuarto año de la carrera, pasó a llamarse "Producción Animal y Calidad de los Productos de Origen Animal". Esta etapa, también conformada por tres módulos, se imparte los trimestres XIII, XIV y XV del quinto y último año de la carrera. De los tres últimos módulos del nuevo plan de estudios, 
es el módulo "Sistemas de Producción Animal" (SPA), impartido durante el segundo trimestre (Primavera) de 2016, por el autor de este artículo, a un grupo de 31 estudiantes inscritos, en que se desarrolló el estudio de caso, materia del mismo artículo.

\section{Fundamentos teóricos sobre los hábitos de estudio y aprendizaje}

Como actividad psíquica, el aprendizaje es una función fundamental de las facultades cerebrales, producto de la transmisión de estímulos quimioeléctricos a través de circuitos neurales eventualmente dispuestos y potencialmente mejorables -con el desarrollo cerebral, durante la infancia y la juventud, y la neuroplasticidad, durante la edad adulta (Nelson, de Haan y Thomas, 2006, pp. 31-33)—, que permite la asimilación, procesamiento, distribución, generación, almacenamiento, recuperación y uso de información por el cerebro mismo. A partir de la actividad psíquica, el aprendizaje trasciende, no bajo las condiciones y circunstancias de eventos aislados, sino a través de la transformación de la realidad de las distintas esferas y entidades sociales sobre las que repercute como actividad formativa (educación, capacitación y experiencia). Así, de acuerdo con la UNESCO (1997, p. 3), el aprendizaje es el mejoramiento del comportamiento, los conocimientos, la comprensión, las actitudes, los valores o las capacidades del sujeto aprendedor. De este modo, las personas gradualmente asimilan información, ideas y valores; obtienen conocimientos teóricos y prácticos y adquieren habilidades y competencias de mayor nivel, que les permiten desenvolverse mejor (Cedefop, 2008, p. 112).

Pudiera decirse que el aprendizaje es sostenible en la medida en que se conserva en la memoria, como resultado sináptico del aprendizaje (LeDoux, 2002, p. 9); y que, asimismo, lo aprendido es reflejo de ese acumulado sináptico. Vistos así, el aprendizaje y la memoria son dos caras de la misma moneda: la sinaptogénesis (formación de sinapsis) entre ambos constructos. En torno al estudio del aprendizaje y la memoria, existen distintas taxonomías de cada uno; sin embargo, para los fines del presente artículo, y en el entendido de que los dos constructos están sinápticamente interconectados, es importante destacar aquí las diferentes formas de aprendizaje y memoria del cerebro humano, de las que se tiene conocimiento hasta ahora, y entre estas, las que están asociadas, de manera general, con la naturaleza de las habilidades y las competencias de las personas.

Sobre la memoria, en general, comúnmente se reconocen tres etapas o estadios: a) la memoria sensorial (inmediata), b) la memoria de trabajo (de corto plazo), y c) la memoria de largo plazo. De la memoria a largo plazo destacan dos tipos: la memoria declarativa o explícita; y la memoria no declarativa o implícita. (Sousa, 2006, p. 81). 
A la memoria declarativa (también llamada explícita o consciente) competen los saberes, expresados a través de un sistema lingüístico determinado, de los que se tiene consciencia por el recuerdo de hechos y cosas aprendidos a lo largo de la vida. Este tipo de memoria se divide a su vez en dos tipos más: la episódica (acontecimientos, hechos, eventos) y la semántica (palabras, objetos, personas, nombres) (Sousa, 2006, p. 81).

Por su parte, las formas no declarativas o implícitas del aprendizaje y la memoria permiten aprender información y habilidades a través de la mera exposición o práctica, sin necesidad de esfuerzo o actividad consciente, llegando eventualmente a "automatizarse" (Nelson et al., 2006, p. 92). De la memoria no declarativa o implícita, Squire, Knowlton y Musen (1993; citados por Nelson et al., 2006, p. 93) identificaron tres formas primarias: primado (priming), aprendizaje procedimental (habilidades y hábitos) y condicionamiento clásico (aprendizaje asociativo), cada uno con su respectivo sistema neural.

\subsection{Aprendizaje procedimental}

Desde el punto de vista neurocientífico, el aprendizaje procedimental, también llamado aprendizaje implícito, aprendizaje de hábitos o aprendizaje de habilidades, supone la adquisición lenta e inconsciente de una base de conocimientos y habilidades conductuales, establecida a través del tiempo, que es difícil si no imposible de explicar (Nelson et al., 2006 p. 94). En el ámbito formativo (educación, capacitación, experiencia), esta base de conocimientos y habilidades conductuales concierne a la adquisición del saber hacer; al desarrollo de las habilidades físicas y mentales necesarias para hacer o conseguir algo, y al dominio de procesos, técnicas, métodos, estrategias y pautas de acción. Por consiguiente, el lado neural del aprendizaje y la memoria procedimental y el lado formativo de las competencias de las personas en el curso de sus vidas, aunque se les aborde por separado, funcionan a la par. En otras palabras, la formación de las competencias de individuos y grupos es interdependiente, en tiempo y forma, de la sinaptogénesis de conocimientos y habilidades conductuales, propias del aprendizaje y la memoria procedimental, pese a que, para fines evaluativos, las competencias se reconozcan "independientemente del cuándo y cómo fueron adquiridas".

\subsection{Sinaptogénesis}

Todas las funciones del cerebro por las que los saberes se aprenden implican las sinapsis nerviosas; es decir, los espacios de proximidad y comunicación funcional entre neuronas. Si los seres humanos en esencia son lo que saben, la tarea de la educación y los 
educadores, consiste en favorecer y aprovechar el potencial genético del desarrollo cerebral —durante la infancia y hasta los 20 años de edad-y la neuroplasticidad adulta — de los 20 años en adelante (Nelson et al., 2006) - mediante experiencias y ambientes de aprendizaje ricos, significativos, estimulantes, de calidad, acordes a cada etapa de la vida y a las inclinaciones y capacidades de cada individuo. Evidentemente, el potencial biológicoformativo del cerebro es mucho más un asunto de posibilidades que de certezas. El cerebro tiene la capacidad de aprender, desaprender, reaprender y cambiarse a sí mismo, comprometiendo y trastocando la formación de sinapsis (sinaptogénesis), y en consecuencia, la configuración de los patrones de los circuitos nerviosos con que funciona.

Según Edelman (1987; citado por LeDoux, 2002 p. 73) las sinapsis en el cerebro de los seres humanos compiten para estar vivas: las que son utilizadas sobreviven, mientras que las que no se utilizan se extinguen. De acuerdo con dicho autor, "el patrón de circuitos neurales no se establece ni reorganiza instructivamente", a partir de indicaciones o reglas de influencias externas, sino que "las influencias externas seleccionan las sinapsis iniciando y reforzando ciertos patrones de actividad neural que las involucran".

\subsection{Habilidades, hábitos y rutinas}

Como se ha hecho ver, las formas del aprendizaje y la memoria procedimental involucran, particularmente, las habilidades físicas e intelectuales de las personas, y estas, a su vez, a las competencias de individuos y grupos en situaciones concretas (económicas, sociales, culturales, tecnológicas). En la medida en que las habilidades -precursores y componentes de competencias - se dominan, su desempeño tiene lugar de manera inconsciente, automática. De tal modo, quien sabe andar en bicicleta, como quien sabe leer o escribir, sabe hacerlo, aunque no pueda explicar cómo. En otro nivel de complejidad, las habilidades manuales de un cirujano, las habilidades del pensamiento lógico de un matemático o las habilidades expresivas de un artista, también se desarrollan de manera inconsciente —aprendizaje no declarativo, implícito-, dentro del dominio de la capacidad reflexiva, pensante -aprendizaje declarativo, explícito-. Las personas competentes son conscientes de las variantes deseadas y no deseadas en la calidad de su trabajo, están muy lejos de ser autómatas, si lo fueran, dejarían de ser competentes.

Para que el cerebro se perfeccione y pueda alcanzar un desempeño "óptimo" debe habituarse al trabajo que él mismo se plantea. Muchas de nuestras actividades cotidianas son -o se convierten en- rutinas para las que requerimos de habilidades intelectuales y físicas forjadas a través de las formas del aprendizaje y la memoria procedimental. A partir 
de estas habilidades se forjan los hábitos, y de los hábitos, las rutinas, que, a su vez, fortalecen los hábitos que las sostienen dentro de la trama nerviosa. Entiéndase por hábitos, los patrones regulares de comportamiento que tienen resultados y efectos sistemáticos, ya sean positivos (buenos hábitos) o negativos (malos hábitos), sobre el individuo y su entorno; y por rutinas, los quehaceres físicos e intelectuales a que nos hemos habituado 0 acostumbrado hasta enraizarlos en nuestro modus operandi, modus vivendi, e incluso, modo de ser. Hábitos y rutinas son manifestaciones significativas de la conducta de los seres humanos a nivel individual y de grupos.

La formación de un hábito supone cambios de conducta sujetos a respuestas neurales de distinto orden, cuya automatización varía de un individuo a otro según el grado de complejidad del hábito en cuestión. Lally et al. (2010) encontraron que el tiempo requerido para la automatización de hábitos simples —como son, por ejemplo, las conductas alimentarias, los hábitos de consumo de agua y la práctica del ejercicio físico-varía considerablemente en un rango de 18 a 254 días, con una mediana de 66 días. Por otra parte, los hallazgos de Verplanken (2006; citado por Lally et al., 2010) sugieren que la automatización de conductas es más rápida y de mayor grado de automaticidad en los hábitos simples que en los complejos. Lo anterior supone que la adquisición de hábitos de estudio y de aprendizaje aplica para lapsos considerablemente mayores a un trimestre académico (12 semanas) y está sujeta a condiciones prolongadas de oportunidad y ejercitación de facultades físicas y mentales.

Mientras las habilidades son neutrales —ni malas ni buenas_y los hábitos pueden ser positivos o negativos, las rutinas funcionan como accionadores - acciones detonadoras- de productos y resultados, de mayor o menor impacto (político, económico, social, cultural, ambiental) a través del tiempo. Desde otra perspectiva, de las rutinas interesa su efectividad; de los hábitos, fomentar los buenos y eliminar los malos; y de las habilidades, el que las personas las adquieran y dominen. La Figura 1 muestra esquemáticamente la interrelación de los factores referidos, dentro de un marco de evaluación que sirva a las partes involucradas (stakeholders) en la formación de personas competentes (psicológica, social y profesionalmente hablando). 
Figura 1

El aprendizaje procedimental y sus interrelaciones causa-efecto con el entorno y desarrollo formativo del sujeto aprendedor

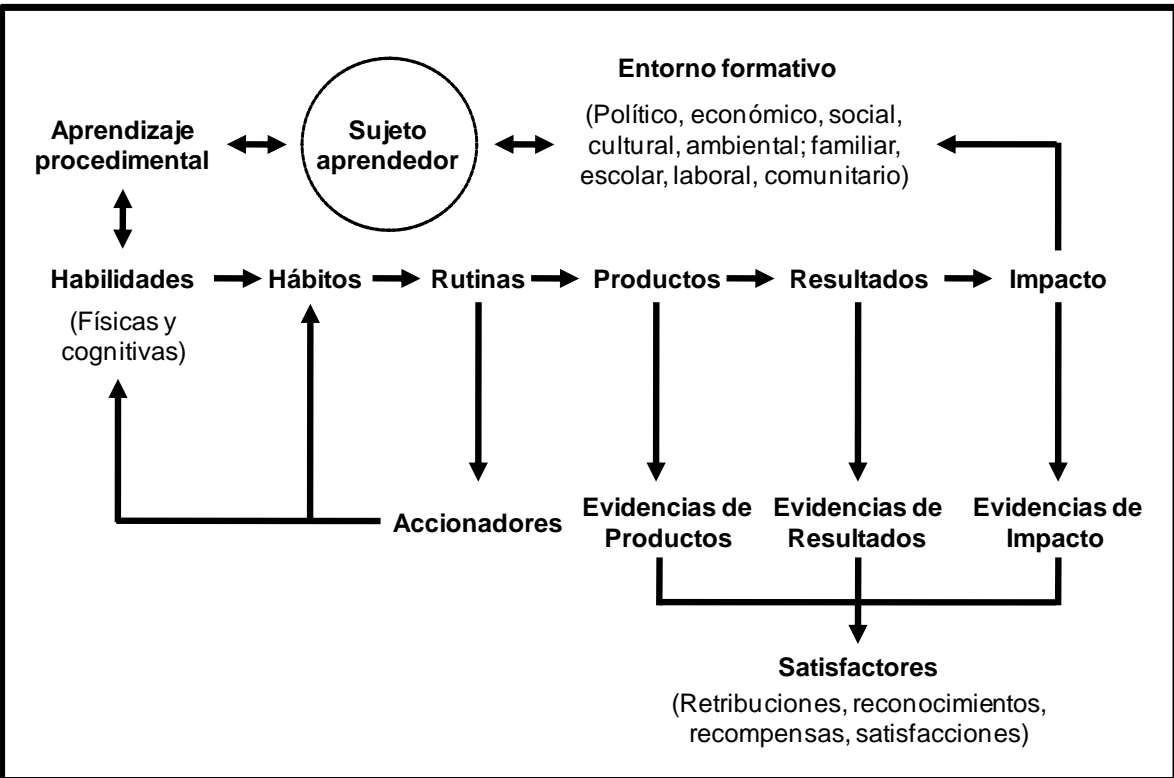

Fuente: Elaboración propia del autor (2017).

Para la educación y las tareas docentes, el aprendizaje de hábitos supone el fomento y la aplicación de las habilidades cognitivas y físicas del educando en tres sentidos fundamentales: a) la adquisición, desarrollo y reforzamiento de buenos hábitos -positivos, valiosos, útiles a los propósitos formativos del individuo-; b) la identificación, prevención y eliminación de malos hábitos —negativos, dañinos, sin utilidad ni provecho-; y c) el cambio de malos hábitos por buenos. Sobre el aprendizaje de hábitos es pertinente considerar las siguientes premisas:

- A lo largo de la vida, las personas adquieren, modifican y desarrollan una serie de patrones de hábitos que, en alguna medida, definen el curso ambispectivo — pasado y futuro- de su propia identidad.

- El individuo hace de sus hábitos — buenos y malos- parte de sí mismo y de lo que, con el tiempo, puede llegar a ser.

- Para sostenerse, los hábitos requieren de rutinas y viceversa, para que las rutinas existan precisan de hábitos.

- La formación y mantenimiento de un hábito supone, en principio, la repetición, reforzamiento y automatización de su práctica, bajo condiciones dadas (Lally et al., 2010). 
- A mayor cantidad y sofisticación de las habilidades implicadas en un hábito, mayor es la dificultad para conseguir su apropiación automatizada y, por consiguiente, mayor el esfuerzo, tiempo y dedicación necesarios para sistematizar su ejercicio.

- El conjunto de hábitos y rutinas de comportamiento se integra al patrón individual (auto-sistematizado) en que cada persona ocupa -0 tiende a ocuparregularmente su tiempo.

- Los hábitos y sus correspondientes rutinas no son gratuitos: demandan tiempo, energía y recursos de distinta índole — -según el caso.

- Los buenos hábitos pueden verse favorecidos: cuando estos se complementan o, por lo menos, no se contraponen; cuando el individuo dispone de suficiente tiempo, condiciones y medios para convertirlos en rutinas (sistematizarlos); y cuando, para quien los adopta y ejerce, los beneficios y satisfactores implicados valen la pena.

- De lo contrario, dicho patrón tiende a desequilibrarse y mermar: los hábitos positivos -y sus respectivas rutinas - entran en conflicto, se atienden unos a expensas de otros; los urgentes pueden desplazar a los importantes; $y$, mientras algunos de estos hábitos se debilitan e incluso pierden, los menos deseados o no deseados encuentran condiciones para fortalecerse y ganar terreno en el patrón individual de hábitos y rutinas.

- Las condiciones a favor o en contra de la formación de buenos hábitos pueden tener un carácter intrínseco cuando las provee el sujeto aprendedor, o extrínseco, cuando las generan terceras personas.

\section{Metodología}

La investigación llevada a cabo es un estudio de caso, enmarcado en el paradigma cualitativo, con carácter exploratorio, descriptivo y explicativo. El uso de herramientas de estadística descriptiva para obtener información del estudiantado, a través de un cuestionario diseñado para tal efecto, sirve de apoyo al trabajo exploratorio.

La situación del estudio se circunscribe a la población de interés: un grupo de 31 estudiantes de educación superior y su entorno —donde viven, estudian, y algunos de ellos trabajan-, durante la impartición del Módulo "Sistemas de Producción Animal", de la carrera de Medicina Veterinaria y Zootecnia, en el trimestre de Primavera de 2016, en la Universidad Autónoma Metropolitana, Unidad Xochimilco, Ciudad de México.

En cuanto a las técnicas de acopio de información, cabe distinguir, primero, la relativa a los contextos del caso mediante la búsqueda de datos sociodemográficos; segundo, el 
registro y el seguimiento del avance programático del curso (módulo) a lo largo del trimestre; tercero, la investigación bibliográfica sobre los fundamentos teóricos de los hábitos de estudio y aprendizaje; y cuarto, el diseño de un cuestionario dirigido al estudiantado para, de manera voluntaria y anónima, conocer su patrón de hábitos cotidianos y la percepción de la propia población estudiantil sobre sus habilidades y desempeño académico. De tal forma, la investigación conjuga, en una discusión analítica relevante a los factores adversos al fomento de hábitos de estudio y aprendizaje, información sobre los contextos del caso, los fundamentos teóricos de los hábitos de estudio y aprendizaje, los eventos relevantes al avance programático del curso y los hallazgos observados a partir del cuestionario.

La identificación de los factores adversos se realizó por dos vías: primero, sobre el avance del programa académico del curso; y segundo, mediante la aplicación al estudiantado, al término del módulo, del cuestionario antes mencionado. Por su parte, mientras el análisis de los datos obtenidos de la aplicación de dicho cuestionario es de carácter estadístico descriptivo, el estudio en conjunto se apega a los principios del paradigma de investigación sistémico/naturalista/cualitativo (Guba y Lincoln, 1983) y, particularmente, a las características, alcances y límites del estudio de casos. Se asume que el estudio de casos, tradicionalmente asociado con la investigación cualitativa, no sigue una metodología estricta, sino una forma de selección coherente de los sujetos u objetos de estudio, donde se presta especial atención a incógnitas que pueden ser esclarecidas a través de situaciones concretas (Buendía, Colás y Hernández, 1998, p. 257).

\section{Resultados}

Los hallazgos de investigación observados corresponden a dos aspectos estrechamente interrelacionados: por un lado, el concerniente a la identificación de factores adversos al fomento de hábitos de estudio y aprendizaje; y por otro, el relativo a los posibles efectos de dichos factores sobre el desempeño académico estudiantil.

\subsection{Identificación de factores adversos}

Como se indicó anteriormente, el problema central del caso radica en la falta de buenos hábitos y rutinas de estudio y aprendizaje para el adecuado aprovechamiento estudiantil bajo las condiciones y circunstancias de la situación analizada. En este sentido, se tienen por factores adversos los elementos que contribuyen a la generación de dicho problema en el ámbito de dos grandes aspectos: el relativo a los hábitos de distinta índole, los cuales compiten con los hábitos de estudio y aprendizaje deseados; y el referente a las 
circunstancias, contingencias e imprevistos, que dificultan u obstaculizan la formación de estudiantes, particularmente en lo relacionado con el aprendizaje procedimental de habilidades físicas y cognitivas de especial interés.

Conforme a lo expuesto en el apartado sobre metodología, la identificación de los factores adversos se realizó por dos vías; primero, a través del avance del programa académico del curso; y segundo, mediante la aplicación al estudiantado, al término del módulo, de un cuestionario para conocer sus actividades rutinarias. Aunque la información se obtuvo en dicho orden, el curso de los eventos del caso ocurrió en sentido opuesto: primero, en referencia a la información vertida a través del cuestionario, y posteriormente, en relación con la información y los acontecimientos registrados durante el programa de actividades académicas del trimestre. Por tal razón, la identificación de los factores adversos se ajustó a esta última pauta: empieza con la información del cuestionario y termina con la sucesión de acontecimientos sobre el avance programático del módulo.

\subsubsection{Actividades rutinarias}

El día del examen final del módulo, conforme el estudiantado entregaba sus pruebas, se les proporcionó un cuestionario de carácter anónimo y confidencial, con el fin de recabar información sobre los hábitos y rutinas en que ocupan su tiempo. Las 30 personas que asistieron al examen resolvieron el cuestionario (ver anexo 1). Esencialmente, el cuestionario se formuló con el propósito de conocer, a partir del punto de vista estudiantil, las actividades habituales a las que conceden mayor prioridad, tiempo e interés, al grado de convertirse en patrones rutinarios, con los que, en cierta forma y medida, atienden sus respectivas necesidades, intereses y obligaciones. A cada estudiante se le solicitó información relacionada con los siete principales quehaceres o actividades habituales en que distribuyen su tiempo (agenda), dentro de las 24 horas del día y los siete días de la semana. Para tal efecto, se plantearon las siguientes 10 opciones:

- Trabajo (remunerativo).

- Tareas domésticas.

- Asistencia a la UAM.

- Estudio.

- Transporte.
- Entretenimiento.

- Deporte.

- Redes sociales.

- TV.

- Otra (especifica). 
Sobre la base de la información obtenida, la Tabla 1 muestra las actividades que forman parte de las rutinas estudiantiles, en consideración a: el número de estudiantes que las declara; el promedio de sesiones a la semana por estudiante; el promedio de horas por sesión, por estudiante; y el promedio de horas dedicadas a cada actividad, por semana y por estudiante.

Tabla 1

Número, porcentaje y razón tiempo/sesión del estudiantado por actividad rutinaria

\begin{tabular}{|l|c|c|c|c|c|}
\hline \multicolumn{1}{|c|}{ Actividad } & $\begin{array}{c}\text { No. de } \\
\text { estudiantes }\end{array}$ & $\%$ & $\begin{array}{c}\text { Sesiones/Semana } \\
\text { /Estudiante }\end{array}$ & $\begin{array}{c}\text { Hrs./Sesión } \\
\text { /Estudiante }\end{array}$ & $\begin{array}{c}\text { Hrs./Semana } \\
\text { /Estudiante }\end{array}$ \\
\hline Trabajo & 20 & 67 & 4.6 & 5.7 & 25.7 \\
\hline Tareas domésticas & 24 & 80 & 4.7 & 1.6 & 6.3 \\
\hline Asistencia a la UAM-X & 30 & 100 & 4.4 & 3.9 & 17.2 \\
\hline Estudio & 27 & 90 & 4.4 & 1.9 & 7.7 \\
\hline Transporte & 30 & 100 & 6.5 & 2.6 & 14.9 \\
\hline Entretenimiento & 20 & 67 & 3.6 & 3.4 & 9.0 \\
\hline Deporte & 12 & 40 & 4.2 & 2.1 & 7.3 \\
\hline Redes sociales & 23 & 77 & 15.1 & 1.3 & 10.2 \\
\hline TV & 7 & 23 & 4.3 & 1.5 & 5.9 \\
\hline Otras & 10 & 33 & 5.3 & 4.2 & 14.5 \\
\hline
\end{tabular}

Fuente: Elaboración propia del autor (2017), a partir de las actividades rutinarias declaradas por el estudiantado del grupo.

De entre las 10 opciones enunciadas — valga reiterar - cada estudiante señaló siete de ellas como las actividades habituales dominantes dentro del patrón de rutinas personal que revela la forma en que regularmente utiliza su tiempo. Con base en los datos de la Tabla 1 , en seguida, se exponen algunos de los aspectos preponderantes sobre los patrones rutinarios del estudiantado del grupo:

Únicamente tres actividades están directamente relacionadas con la formación académico-formativa estudiantil: asistencia a la Universidad, estudio y deporte. La primera, la declara todo el estudiantado (100\%), la segunda casi todo (90\%) y la tercera, arriba de la tercera parte (40\%). Si bien, para las primeras dos actividades, el estudiantado destina el mismo número de sesiones (4.4) a la semana, para la asistencia a la Universidad ocupan un promedio de 17.2 horas a la semana, mientras para el estudio (lecturas, tareas, trabajo de investigación), solo 7.7 horas por semana.

Dado que el estudiantado tuvo cuatro sesiones de clase a la semana, cada una de no más de 4 horas, el límite de tiempo en el salón de clases fue de 16 horas a la semana. Los viernes se dedicaron a la realización de ejercicios prácticos por equipos, en distintas unidades de producción animal, para lo que requerían entre 4 y 6 horas. De este modo, la 
razón horas de estudio: horas de clase (7.7/16) por semana, es de .48:1, cuando debería ser, por lo menos, de 1 a 2 horas de estudio por hora de clase en estudiantes de tiempo completo (40 horas a la semana) (Universia, 2015). Es pertinente hacer notar que el 10\% del estudiantado del grupo no consideró al estudio como actividad habitual. El 40\% que dice practicar deporte (dentro o fuera de las instalaciones de la Universidad) realiza alrededor de 4.2 sesiones de ejercicio físico por semana, con un promedio de 2.1 horas por sesión y 7.3 horas a la semana; es decir, incluso por debajo del tiempo (7.7 horas) destinado al estudio a la semana. El resto (60\%) no práctica ningún deporte de manera regular.

A diferencia de las actividades académico-formativas _asistencia a la Universidad, estudio y deporte_, las demás actividades enunciadas no son consistentes con el desarrollo individual ni colectivo de hábitos constructivos de estudio y aprendizaje. Más aún, tres de estas actividades - trabajo, transporte y redes sociales ${ }^{2}$-, en particular, representan factores adversos, debido al elevado número de estudiantes involucrados y la gran cantidad de sesiones y tiempo que invierten en ellas. Dos terceras partes (67\%) del estudiantado del grupo trabajan. Estas personas refieren que a sus actividades laborales (remuneradas) destinan un promedio de 4.6 días a la semana, 5.7 horas por día y 25.7 horas a la semana. Así, el trabajo, como actividad habitual de dos terceras partes del grupo (67\%), consume a la semana un promedio de 8.5 horas más que el promedio de horas (17.2) utilizado a la semana por todo el grupo para asistir a la Universidad. Tal diferencia representa cerca de un $50 \%$ de tiempo más para trabajar que para asistir a la Universidad.

En lo que corresponde al transporte, segundo factor adverso relevante al problema del caso, el 100\% del estudiantado realiza esta actividad (de ida y vuelta) un promedio de 6.5 veces por semana. Cada una de ellas les toma alrededor de 2.6 horas, y en conjunto, semanalmente, un promedio de 14.9 horas. La persona que menos tiempo dedica a transportarse emplea 4 horas a la semana, y la que más, 33. El estudiantado ocupa en su transporte semanal el equivalente a más del $86 \%$ del tiempo promedio que pasan semanalmente en la Universidad, lo cual es por demás significativo para considerar esta actividad como factor adverso.

La participación en redes sociales, como actividad habitual de los patrones rutinarios del alumnado, es el tercer factor adverso más importante de los identificados a partir de la información del cuestionario. Más de tres cuartas partes (77\%) del grupo incluye esta

2 La rutina de estudio está claramente señalada en el cuestionario, por lo que el uso de las redes sociales (Facebook, Whatsapp, Linkedin, Twitter, etc.) no alude a tal fin. 
actividad en su patrón de rutinas, con un promedio de 15.1 sesiones a la semana, 1.3 horas por sesión y 10.2 horas semanales. Los rangos de participación son muy amplios: de 4 a 100 sesiones a la semana, de 10 minutos a 4 horas por sesión, y de 3 a 20 horas a la semana. Dentro de los patrones de rutinas, el promedio de horas dedicado semanalmente a las redes sociales (10.2 horas) es superior al destinado al entretenimiento (9 horas), al estudio (7.7 horas), al deporte (7.3 horas) y a las tareas domésticas (6.3 horas).

\subsubsection{Avance programático}

Para el trimestre de 2016-Primavera se calendarizaron 52 días de clases, del 9 de mayo al 27 de julio del mismo año, y cinco días para evaluaciones globales. Así, el trimestre comprendía 11 semanas de clases y una de evaluación global. En lo que respecta a la impartición del módulo Sistemas de Producción Animal, el profesor destinó cuatro días para sesiones teóricas en el salón de clases, de lunes a jueves, de 12:00 a 16:00hrs, y un día, los viernes, para la realización de prácticas y tareas de investigación modular en distintas unidades de producción, como granjas avícolas, porcinas y cunícolas, y explotaciones lecheras. Al inicio de clases (lunes 9 de mayo), se tenía una lista de 26 estudiantes asignados al grupo. Durante la primera semana, en el estudiantado puede tramitar cambios de grupo, se dieron de baja 7 y de alta 12, de modo que el grupo quedó conformado por 31 estudiantes, cinco más de los inicialmente asignados.

El primer día de clases, el docente proporcionó información al grupo — de manera verbal y por escrito- sobre los objetivos, el contenido temático, las actividades académicas y los requisitos para la acreditación del módulo; asimismo, el estudiantado externó sus intereses, expectativas, observaciones, comentarios y dudas. En esa misma sesión, al estudiantado se le hizo entrega de la Guía de Operación Modular (syllabus), ${ }^{3}$ documento de 25 páginas cuyos principales apartados son los siguientes:

- Datos de identificación del módulo (nombre, clave, grupo, docente, trimestre, aula, horario).

- Objetivos (general y específicos).

- Índice temático (semana, tema/unidad, objetivo, contenido, actividades académicas).

\footnotetext{
${ }^{3}$ El formato para la elaboración de la Guía de Operación Modular es proporcionado por la División de Ciencias Biológicas y de la Salud, de la UAM-Xochimilco.
} 
- Evaluación (exámenes escritos, estudio de factibilidad de proyecto, seminarios, maqueta, reportes de prácticas).

- Bibliografía (general y específica, por semana).

- Cronograma y programación de actividades (semana, día, hora, tema/actividad, lugar, responsable, observaciones).

En esencia, la Guía de Operación Modular (GOM) es una descripción pormenorizada del contenido del módulo (curso) en cuanto a objetivos, temas, tiempos, actividades y métodos de enseñanza-aprendizaje. Sin embargo, más allá de su uso convencional, para el ajuste programático del plan de estudios al calendario escolar y los lineamientos de planeación, desarrollo y evaluación del módulo, este documento sirve como constancia y recordatorio explícito de las tareas, compromisos y responsabilidades asumidos por los integrantes del grupo —docente y estudiantes- para el debido aprovechamiento y la consecución concertada del curso.

Una primera condición para cumplir con los compromisos acordados, en general, y fomentar los hábitos de estudio y aprendizaje del alumnado, en particular, es la asistencia regular y puntual del estudiantado a todas y cada una de las sesiones programadas en la GOM. En relación con dicha condición se registró una serie de eventos en contra: a) impuntualidad, b) inasistencia, c) paros y d) periodo vacacional pos paros. En mayor o menor medida, estos eventos afectaron las rutinas a favor del estudio y el aprendizaje continuo; por consiguiente, atañen directamente al problema del caso.

\subsubsection{Impuntualidad}

Del primer día de labores hasta la fecha del examen final, todos los días se pasó lista al inicio de cada sesión, salvo un día (el 9 de junio) en que el transporte público se complicó más allá de lo usual. Considerando las dificultades que varias personas del estudiantado externaron para asistir puntualmente a la universidad, siempre se les permitió la entrada al salón de clases, pese a que en ocasiones el retraso fue de más de una hora. Durante las 33 fechas en que se pasó lista se registraron 76 tardías, con un promedio de 2.3 por día y un rango de 0 a 6 por día. Las tardías por estudiante promediaron cerca de 2.5 por día y estuvieron en el rango de 0 a 10 durante el período de clases del que se tuvo registro. 


\subsubsection{Inasistencia}

El problema de inasistencia fue considerablemente mayor al de impuntualidad. En los 33 días en que se pasó lista faltaron 143 estudiantes, un promedio de 4.3 por día, con un rango de faltas de 0 a 13 (el viernes 29 de julio). Solo un estudiante no faltó a ninguna de las sesiones de clase. Los que más faltaron fueron: un estudiante, con 13 faltas; 3 estudiantes con 9 faltas cada uno; y 5 estudiantes, con 7 faltas cada uno.

De un total de 1,023 posibles asistencias (de 31 estudiantes en 33 días efectivos de clases), cerca del $14 \%$ fueron faltas, y de las asistencias efectivas (880), el $8.6 \%$ fueron tardías. Así, la suma de faltas (143) y tardías (76) representa el 21.4\% —más de la quinta parte- del total de asistencias posibles.

\subsubsection{Paros}

En apoyo al movimiento magisterial de la Coordinadora Nacional de Trabajadores de la Educación (CNTE), la Asamblea Estudiantil de la UAM-Xochimilco realizó un paro de actividades durante tres días, de la mañana del 22 de junio a las 14:50 del 24 junio. Tres días después, en respaldo a la misma causa, el Sindicato Independiente de Trabajadores de la Universidad Autónoma Metropolitana (SITUAM) efectuó otro paro de labores, entonces, por 24 horas, de las 22:00 horas del lunes 27 a las 22:00 horas del martes 28 de junio (Hernández, 2016). El 29 de junio se restablecieron las actividades normales, que continuaron así hasta el 6 de julio, tan solo seis días. El jueves 7 de julio la Asamblea Estudiantil vuelve al paro como medida de presión a diferentes demandas, con lo que la suspensión de actividades, que dejó sin clases a más de 15,000 estudiantes del plantel Xochimilco, se prolongó durante tres semanas más, del 7 al 27 de julio. De esta forma, la UAM-Xochimilco estuvo cerrada durante 19 días hábiles del programa académico inicial.

\subsubsection{Periodo vacacional posparos}

Tras conjurarse el último paro, el miércoles 27 de julio el Colegio Académico —órgano integrado por representantes de todos los sectores de la UAM, que rige la vida institucional de la Universidad- en sesión urgente, aprobó la recalendarización de las actividades académicas. Con los ajustes aprobados al calendario escolar, la Unidad-Xochimilco laboró de manera continua solo dos días más —el jueves 28 y el viernes 29 de julio-, ya que el lunes 1 de agosto las actividades volvieron a suspenderse, entonces por el inicio del periodo vacacional, de cuatro semanas, que se mantuvo sin modificaciones, conforme al calendario 
original, hasta concluir el domingo 28 de agosto. El lunes siguiente, 29 de agosto, se reanudaron las clases, tan solo por dos semanas más, a efecto de concluir el trimestre clases, evaluación global y entrega de notas finales- antes del 9 de septiembre (Sánchez y Arellano, 2016).

\subsection{Desempeño académico}

El fomento de habilidades, hábitos y rutinas de estudio y aprendizaje, de progresiva complejidad y nivel, en el curso de la formación de individuos, grupos y generaciones de estudiantes, responde, por una parte, a la calidad de la educación proporcionada, y por otra, al desempeño académico estudiantil. De ahí que, para mejorar la calidad de la educación superior, sea materia obligada la sistematización — desarrollo de ciclos virtuosos- de habilidades, hábitos y rutinas de estudio y aprendizaje, en consideración con los factores adversos al desempeño académico del alumnado.

Se asume que los factores adversos identificados en este estudio atañen, en distinta forma y medida, al curso ambispectivo - pasado y futuro- de la formación profesional del estudiantado del grupo. A lo que, las tres últimas preguntas, de las cuatro formuladas a través del cuestionario, proporcionan información sobre aspectos relevantes al desempeño académico alcanzado por el estudiantado hacia la conclusión de la carrera. Dichos aspectos son: a) habilidades esenciales; b) fuentes de información; c) módulos cursados y reprobados y d) promedio de calificaciones. A continuación, se aborda cada uno de ellos.

\subsection{Habilidades esenciales}

Si bien, a las habilidades y las competencias individuales y de grupos se les refiere desde una amplia variedad de taxonomías, tipos y categorías, para los propósitos del presente caso, es conveniente examinar algunas de las habilidades que son precursoras y componentes esenciales de las competencias académicas esperadas en el ingreso, transcurso y egreso estudiantil de educación superior, en general, y de la carrera de Medicina Veterinaria y Zootecnia que se imparte en la UAM-Xochimilco, en particular. Bajo ciertos esquemas, a algunas de estas habilidades se les denomina "claves" (key skills) y bajo otros, "funcionales" (functional skills); sin embargo, independientemente del nombre que se les asigne, son fundamentales para la formación de un sinnúmero de competencias académicas y profesionales. 
Las habilidades mencionadas, llamadas aquí "esenciales", son imprescindibles para la construcción de configurados complejos — ciclos virtuosos_ entre habilidades, hábitos y rutinas de estudio y aprendizaje, cuyas inconsistencias se reflejan en el problema central del caso: la falta de buenos hábitos y rutinas de estudio y aprendizaje para el adecuado aprovechamiento estudiantil en el contexto del módulo de enseñanza-aprendizaje abordado. Con el objeto de conocer la percepción estudiantil sobre el nivel de sus habilidades esenciales, al cuestionario que en su oportunidad contestaron (ver Anexo 1), se le incluyó una pregunta relativa a la calificación que le asignan a cada una de siete de estas habilidades en una escala de 1 a 5 , donde 1 es muy malo; 2 , malo; 3 , regular; 4 , bueno; y 5 , muy bueno.

La Tabla 2 sintetiza la apreciación del estudiantado, en conjunto, sobre el nivel que poseen en cada una las siete habilidades consideradas. Como puede observarse, el promedio de calificación es regular para matemáticas y escritura; de regular a bueno, en comunicación, trabajo en equipo y lectura; y bueno, con respecto a TIC y creatividad. Así pues, sus mayores deficiencias las perciben en matemáticas y escritura; confían en sus habilidades de lectura, comunicación y trabajo en equipo; y piensan que son bastante buenos en cuanto a TIC y creatividad.

Tabla 2

Estadísticas descriptivas sobre la percepción estudiantil del nivel que poseen en distintas habilidades esenciales

\begin{tabular}{|l|c|c|c|c|c|c|c|}
\hline \multicolumn{1}{|c|}{ Habilidad } & Lectura & Escritura & Comunicación & $\begin{array}{c}\text { Matemáti } \\
\text { cas }\end{array}$ & Creatividad & $\begin{array}{c}\text { Trabajo } \\
\text { en } \\
\text { equipo }\end{array}$ & TIC \\
\hline Promedio/grupo & 3.7 & 3.3 & 3.5 & 3.1 & 4.1 & 3.6 & 3.9 \\
\hline $\begin{array}{l}\text { Desviación } \\
\text { estándar }\end{array}$ & 0.60 & 0.84 & 0.78 & 0.98 & 0.82 & 0.81 & $\begin{array}{c}0.6 \\
6\end{array}$ \\
\hline Mediana & 4.0 & 3.0 & 3.0 & 3.0 & 4.0 & 4.0 & 4.0 \\
\hline Moda & 4 & 4 & 3 & 3 & 4 & 4 & 4 \\
\hline $\mathrm{N}$ & 30 & 30 & 30 & 30 & 30 & 30 & 30 \\
\hline
\end{tabular}

Fuente: Elaboración propia del autor (2017), con base en la información recopilada mediante el cuestionario.

Adicionalmente, la Figura 2 proporciona información sobre el porcentaje de estudiantes en los diferentes niveles ( 1 a 5 ) de calificación, bajo cada una de las habilidades esenciales observadas. De ello, interesa resaltar los siguientes aspectos:

- Solo en lectura y TIC no hay estudiantes con calificaciones de 1 ("muy malo") y 2 ("malo"). En todos los demás conceptos existen estudiantes que declaran poseer niveles deficientes de habilidad. 
- Para efectos académicos y profesionales, merece especial atención la existencia de estudiantes que se tienen por "muy malos" o "malos" en comunicación $(6.7 \%$ de ellos), escritura (13.3\%), creatividad (20\%), matemáticas (23.4\%) y trabajo en equipo (43.4\%), en esta última, de forma notoria.

- En todas las habilidades, las calificaciones predominantes se registran en los niveles 3 y 4; es decir, de "regular" a "bueno".

- Si bien, en cinco de las siete habilidades hay estudiantes que se precian de ser "muy buenos" (nivel 5), el porcentaje es bajo, no rebasa al 10\%, a excepción de las TIC, con menos de la quinta parte del grupo (16.7\%).

- Sin duda, la habilidad estimada con mayores niveles de dominio es TIC. No se registran estudiantes con niveles bajos ( 1 y 2 ); poco más de la cuarta parte se califica como "regular" (nivel 3) y el resto, cerca de tres cuartas partes (73.4\%), se considera "bueno" o "muy bueno".

Figura 2

Porcentaje de estudiantes por calificación (1 a 5) para cada habilidad autoevaluada

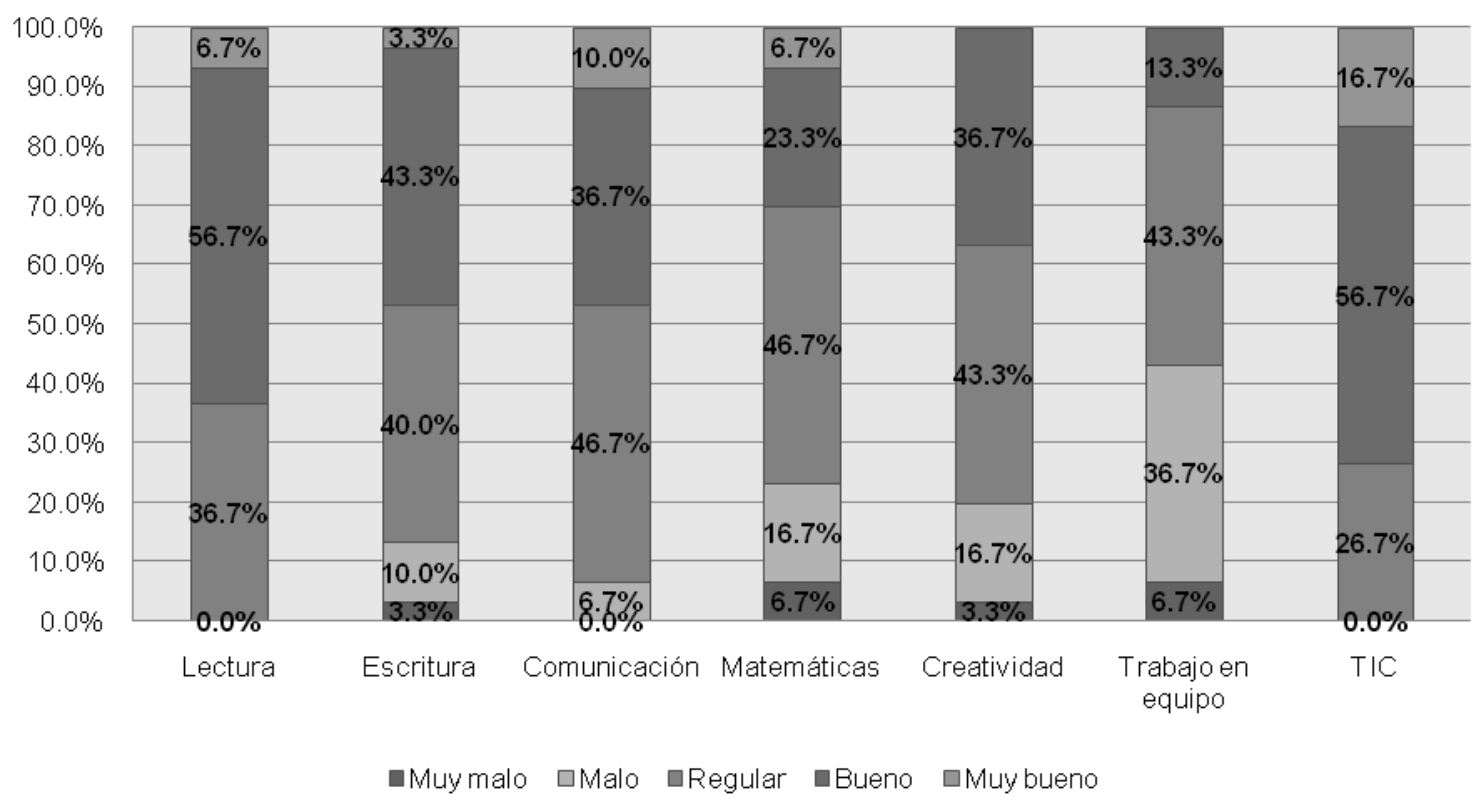

Fuente: Elaboración propia del autor (2017), con base en la información recabada a través del cuestionario.

En el contexto de los resultados obtenidos, valdría afirmar que los factores adversos identificados, lejos de favorecer el mantenimiento y desarrollo sostenido de las habilidades esenciales, entorpecen su incorporación a círculos virtuosos de hábitos y rutinas de estudio y aprendizaje efectivos, con lo que se perjudica el proceso de sinaptogénesis concomitante al 
aprendizaje y la memoria procedimental de habilidades y competencias formativas (claves, funcionales, generales, específicas).

\subsubsection{Fuentes de información}

En lo relativo al desempeño académico estudiantil, otro aspecto de sumo interés son las fuentes de información de las que usualmente se valen como material de estudio. A partir de las respuestas a la tercera pregunta del cuestionario, la Tabla 3 presenta las inclinaciones de los integrantes del grupo, en relación con diversos medios de documentación y estudio, a decir del propio estudiantado.

Tabla 3

Número y porcentaje de estudiantes por medio de documentación y estudio utilizado

\begin{tabular}{|c|c|c|c|c|c|c|c|c|}
\hline Fuente & Internet & $\begin{array}{c}\text { Materiales } \\
\text { proporcio } \\
\text { nados por } \\
\text { el docente }\end{array}$ & $\begin{array}{c}\text { Libros } \\
\text { especializ } \\
\text { ados }\end{array}$ & $\begin{array}{c}\text { Revistas } \\
\text { científicas }\end{array}$ & $\begin{array}{c}\text { Notas } \\
\text { de } \\
\text { clase }\end{array}$ & $\begin{array}{c}\text { Artículos } \\
\text { científicos } \\
\text { (Biblioteca) }\end{array}$ & $\begin{array}{c}\text { Apuntes } \\
\text { de } \\
\text { compañ } \\
\text { eros }\end{array}$ & $\begin{array}{c}\text { Artículos } \\
\text { técnicos } \\
\text { (Biblioteca } \\
\text { ) }\end{array}$ \\
\hline $\begin{array}{c}\text { No. de } \\
\text { estudiant } \\
\text { es }\end{array}$ & 18 & 16 & 15 & 7 & 7 & 6 & 0 & 0 \\
\hline $\begin{array}{c}\% \text { de } \\
\text { estudiant } \\
\text { es }\end{array}$ & 60.0 & 53.3 & 50.0 & 23.3 & 23.3 & 20.0 & 0.0 & 0.0 \\
\hline
\end{tabular}

Fuente: Elaboración propia del autor (2017), a partir de la información vertida en el cuestionario.

De acuerdo con las respuestas recabadas, los tres principales medios fueron: primero, Internet (60.0\%); segundo, materiales proporcionados por el docente (53.3\%); y tercero, libros especializados (50.0\%). Menos de la cuarta parte del estudiantado incluye las revistas científicas (23.3\%), las notas de clase (23.3\%) y los artículos científicos (20.0\%); y ninguno, los apuntes de compañeros y los artículos técnicos. Sin embargo, arriba del $40 \%$ del estudiantado se pronuncia por las revistas científicas o los artículos científicos. La preferencia por Internet podría asociarse con las señaladas habilidades del alumnado en el manejo de las TIC, así como con la accesibilidad, versatilidad, facilidad y bajo costo que brinda la Red. No obstante, el estudiantado que utiliza esta vía pueden limitarse a buscar, copiar y pegar textos e imágenes, sin llegar a aprovecharla para verdaderos o mayores propósitos de estudio.

Los materiales proporcionados por el docente facilitan las tareas de enseñanzaaprendizaje, sobre todo para estudiantes que no toman notas, prácticamente más de tres cuartas partes del grupo (76.7\%); sin embargo, aun cuando los materiales facilitados por el docente son un complemento de la información de otras fuentes, las notas de cada 
estudiante — sobre distintos temas, fuentes de información y modalidades de enseñanzaaprendizaje - tienen un valor didáctico irremplazable.

Es probable que la poca disposición para tomar apuntes esté relacionada con: a) la falta de habilidad para escribir — de "muy mala" a "regular" en más de la mitad (53.3\%) del estudiantado-; b) el trabajo y la concentración que implica sintetizar información e ideas sobre los diversos contenidos temáticos del curso -bajo diferentes técnicas y recursos didácticos-; y c) la facilidad y bajo costo (tiempo, dinero y esfuerzo) de los medios informáticos. Así, mientras el uso de las TIC puede servir para contrarrestar algunos de los efectos negativos de los factores adversos identificados en el estudio, también puede contribuir a agravarlos, e incluso, a reducir el ejercicio y la sinaptogénesis de facultades cerebrales complejas, como el aprendizaje y la memoria procedimental.

\subsubsection{Módulos cursados y reprobados, y promedio de calificaciones}

Cuanto mejores sean los hábitos de estudio y aprendizaje (más frecuentes, efectivos y duraderos), mejor será el desempeño académico estudiantil. La cuarta y última pregunta del cuestionario, relativa a este supuesto, aporta información, sintetizada en la Tabla 4, sobre las calificaciones obtenidas por el estudiantado del grupo en el transcurso de la carrera, ya próximos a concluirla. Según los datos proporcionados por el estudiantado, solo la quinta parte de esta población (6 de 30) no había reprobado ningún módulo; dos quintas partes (12 de 30) habían reprobado entre un módulo y menos del 10\% de los módulos estudiados; y las dos quintas partes restantes, habían sido suspendidos en más del 10\% de los módulos hasta entonces cursados. Asimismo, de estos tres grupos, el primero tenía un promedio de calificaciones más alto (8.3 de 10) que el de los otros dos (7.6 de 10).

\section{Tabla 4}

Estadísticas descriptivas del promedio de calificación acumulado durante la carrera, en general, y por porcentaje de módulos reprobados

\begin{tabular}{|l|c|c|c|c|c|c|}
\hline $\begin{array}{l}\text { Grupo por \% de módulos } \\
\text { reprobados en la carrera }\end{array}$ & $\mathbf{N}$ & Media & Mediana & Mínimo & Máximo & $\begin{array}{c}\text { Desviación } \\
\text { estándar }\end{array}$ \\
\hline Ningún módulo reprobado & 6 & 8.3 & 8.0 & 7.8 & 9.3 & 0.567 \\
\hline $\begin{array}{l}\text { Al menos 1 y menos de 10\% } \\
\text { de módulos reprobados }\end{array}$ & 12 & 7.6 & 7.6 & 7.0 & 8.2 & 0.472 \\
\hline $\begin{array}{l}\text { Más del 10\% de módulos } \\
\text { reprobados }\end{array}$ & 12 & 7.6 & 7.5 & 7.0 & 8.3 & 0.423 \\
\hline Total & $\mathbf{3 0}$ & $\mathbf{7 . 7}$ & $\mathbf{7 . 8}$ & $\mathbf{7 . 0}$ & $\mathbf{9 . 3}$ & 0.543 \\
\hline
\end{tabular}

Fuente: Elaboración propia del autor (2017), con base en la información recabada a través del cuestionario. 
De tal forma, en las postrimerías de la carrera, el desempeño académico de más del $80 \%$ del estudiantado del grupo era de regular a bajo, con 8 o menos de promedio de calificaciones, y/o uno o más módulos reprobados. El porcentaje general de reprobación fue de $11.6 \%$ de los módulos cursados.

\section{Conclusiones}

La adquisición y desarrollo de buenos hábitos de estudio y aprendizaje es una precondición para el mejoramiento de la educación, en general, y de la educación superior, en particular. Esta precondición, sin que siempre se cumpla, usualmente se da por hecho en el diseño de políticas, iniciativas e instrumentos para elevar el aprovechamiento estudiantil en una amplia gama de esferas: desde los contextos geopolíticos -internacionales, regionales, nacionales, locales - hasta los espacios propios de la actividad docente instituciones educativas, medios académicos, aulas. El estudio de caso, materia del presente artículo, atiende a dicha precondición a partir del planteamiento de un problema central: la falta de buenos hábitos y rutinas de estudio y aprendizaje para el adecuado aprovechamiento académico de un grupo de 31 estudiantes, durante la impartición del módulo (curso) "Sistemas de Producción Animal", de mayo a julio de 2016, dentro del programa de la Licenciatura en Medicina Veterinaria y Zootecnia, que ofrece la Universidad Autónoma Metropolitana, Unidad Xochimilco, en la Ciudad de México.

Para un desempeño académico progresivo, a la altura de los requerimientos de la educación superior, es preciso que el estudiantado disponga de buenos hábitos de estudio y aprendizaje; es decir, de patrones de estudio y aprendizaje regulares con resultados y efectos sistemáticos sobre su desarrollo personal y el de su entorno (político, social, cultural, económico, medioambiental). En este sentido, las habilidades físicas y cognitivas, los hábitos y las rutinas del individuo se interrelacionan e interactúan, de manera sostenida, como elementos de complejos entramados neurales, especialmente en lo concerniente a la memoria no declarativa o implícita y el aprendizaje procedimental, estrechamente asociados con el desarrollo de competencias académicas y profesionales.

Cuando el estudiantado dispone de las habilidades esenciales para realizar estudios de licenciatura —específicamente en Medicina Veterinaria y Zootecnia en la UAM, Xochimilcopero carece de los hábitos de estudio y aprendizaje, acordes a las demandas de su formación profesional, se requiere de lapsos superiores a los de un curso (trimestre) académico para adquirirlos y consolidarlos, puesto que se trata de hábitos complejos y no de 
simples. De lo contrario, cuando el estudiantado no cuenta con las habilidades esenciales al nivel adecuado, el empeño en la construcción de hábitos debe empezar por la atención de estas habilidades. Afrontar ambas problemáticas implica la instauración de medidas correctivas y mecanismos de evaluación y seguimiento a lo largo de toda la carrera.

Contra la eventual adquisición de buenos hábitos de estudio y aprendizaje, existen de facto o potencialmente- innumerables factores adversos. A través del estudio realizado se identificaron seis de ellos, relevantes al contexto y las circunstancias específicas del caso. Tres en el carácter de hábitos y rutinas que compiten con los hábitos de estudio y aprendizaje deseados: a) trabajo, b) transporte y c) redes sociales; y tres más, producto de las circunstancias, contingencias e imprevistos que dificultan u obstaculizan la formación de estudiantes, especialmente en aspectos concernientes al aprendizaje procedimental de habilidades físicas y cognitivas: a) impuntualidad, b) inasistencia y c) interrupción prolongada del programa de actividades académicas preestablecido.

Dentro del extenso escenario de posibles factores adversos, los identificados en el estudio discurren a través de cuatro contextos: a) el global —las redes sociales vinculadas a las TIC—, b) el poblacional y urbano — la necesidad de trabajo (ingresos) y las dificultades de transporte-, c) el institucional —la interrupción recurrente del programa de actividades académicas - y d) el docente —la impuntualidad e inasistencia del alumnado-. En la interrelación de dichos contextos existe un influjo de los factores adversos observados, de los dos primeros, externos al ámbito académico —el global y el poblacional y urbano_, a los dos últimos, internos al ámbito académico —el institucional y el docente.

Los efectos negativos de los factores adversos identificados, sobre los círculos virtuosos entre habilidades, hábitos y rutinas, impactan el proceso constructivo de la educación en dos aspectos torales: primero, al mermar la calidad de la formación del sujeto discente, desde sus habilidades esenciales hasta sus competencias profesionales, que incluyen metacompetencias; y segundo, como consecuencia del anterior, en menoscabo de las contribuciones del propio sujeto durante su vida productiva.

Invertir en mejoras y esfuerzos para elevar el nivel, la calidad y la capacidad de inclusión de la educación superior en poblaciones objetivo no se limita, pues, a los rubros estrictamente académicos —docencia, investigación, difusión de la cultura—. La atención a los llamados aquí factores adversos es también una tarea clave. En lo relativo al caso del estudio que nos ocupa, entre los factores que generan condiciones perniciosas en detrimento del aprovechamiento escolar, se observó: la vulnerabilidad de la Universidad a 
conflictos de distinto orden y alcance, tanto internos como externos; los graves problemas de movilidad en la Ciudad de México; el elevado número de horas que mucho del alumnado dedica a trabajar para solventar gastos personales y familiares; y el uso excesivo de las redes sociales para fines de entretenimiento y distracción.

Las deficiencias en habilidades esenciales (claves, funcionales, nucleares) reportadas por el estudiantado a través de su autoevaluación, conciernen a la problemática ambispectiva de la educación superior, así como a los factores adversos que, en mayor o menor medida, las propician.

Para elevar el aprovechamiento estudiantil de educación superior en el ámbito del estudio de caso realizado, como también, posiblemente, en situaciones con problemáticas y condiciones similares a las aquí expuestas, es necesario el desarrollo sostenido de las habilidades esenciales, sobre la evidencia probada de su dominio, a lo largo de todo el programa de estudios, puesto que dichas habilidades se corresponden estrechamente con los hábitos de estudio y aprendizaje, cuya automatización requiere de meses -cuando no de años- de reforzamiento y trabajo regular. No está de más subrayar que las habilidades y los hábitos referidos son fundamentales para que el estudiantado consiga una preparación sólida, en aras de mayores y mejores oportunidades de desarrollo personal y profesional.

\section{Referencias}

Buendía, Leonor, Colás, Pilar y Hernández, Fuensanta. (1998). Métodos de Investigación en Psicopedagogía. España: McGraw-Hill/Interamericana de España.

Cedefop. European Centre for the Development of Vocational Training. (2008). Terminology of European Education and Training Policy. A Selection of 100 Key Terms. Luxenburgo: Publications Office of the European Union. Recuperado de http://www.cedefop.europa.eu/files/4064 en.pdf

Climént Bonilla, Juan. (2011). Formación por competencias. Tesauro de términos conceptos e instrumentos. México: Trillas.

Congreso de los Estados Unidos Mexicanos. (1973). Ley orgánica de la Universidad Autónoma Metropolitana. Recuperado de https://www.uam.mx/legislacion/lo/2/\#zoom=z

Del Valle, Sonia. (31 de octubre de 2016). Arrecian las crisis en universidades. Periódico Reforma, Sección Nacional. Recuperado de https://www.reforma.com/aplicacioneslibre/preacceso/articulo/default.aspx?id=974494\&u rlredirect=https://www.reforma.com/aplicaciones/articulo/default.aspx?id=974494 
Guba, Egon y Lincoln, Yvonna. (1983). Epistemological and methodological bases of naturalistic inquiry. En George Madaus y Daniel Stufflebeam (Eds.), Evaluation models: Viewpoints on educational and human services evaluation (pp. 363-382). Massachusetts: Kluwer-Nijhoff.

Hernández, Lilian. (28 de junio de 2016). Sindicato de la UAM realiza paro de 24 horas. Periódico Excélsior. Recuperado http://www.excelsior.com.mx/comunidad/2016/06/28/1101527

Instituto Nacional de Ecología y Cambio Climático (INECC), México. (2011). Cuarto Almanaque de Datos y Tendencias de la Calidad del Aire en 20 Ciudades Mexicanas (2000-2009). México: INECC. Recuperado de http://www2.inecc.gob.mx/publicaciones/libros/652/vallemexico.pdf

Instituto Nacional de Estadística y Geografía (INEGI), México. (2010). Censo de Población y Vivienda 2010. México: INEGI. Recuperado de http://www.inegi.org.mx/sistemas/olap/Proyectos/bd/censos/cpv2010/PT.asp?s=est\&c= 27770\&proy=cpv10 pt\#

Instituto Nacional de Estadística y Geografía (INEGI), México. (2015). Información por Entidad. Dinámica de Crecimiento de la Ciudad de México. México: INEGI. Recuperado de http://cuentame.inegi.org.mx/monografias/informacion/df/poblacion/dinamica.aspx?tema $=\mathrm{me}$

Lally, Phillippa, van Jaarsveld, Cornelia, Potts, Henry y Wardle, Jane. (2010). How are habits formed: Modelling habit formation in the real world. European Journal of Social Psychology, 40(6), 998-1009. Doi: 10.1002/ejsp.674.

LeDoux, Joseph. (2002). Synaptic self. How our brains become who we are. Nueva York: Penguin Books.

Nelson, Charles, de Haan, Michelle y Thomas, Kathleen. (2006). Neuroscience of cognitive development. The role of experience and the developing brain. Nueva Jersey: John Wiley \& Sons.

Organización de las Naciones Unidas para la Educación, la Ciencia y la Cultura (UNESCO). (1997). Clasificación Internacional Normalizada de la Educación (CINE). Recuperado de http://unesdoc.unesco.org/images/0011/001113/111387s.pdf

Sánchez Jiménez, Arturo y Arellano García, César. (27 de julio de 2016). UAM modifica calendario para no perder el trimestre. La Jornada en línea. Recuperado de http://lajornadasanluis.com.mx/nacional/uam-modifica-calendario-no-perder-trimestre/

Sousa, David. (2006). How the brain learns. California: Corwin Press.

Taylor Preciado, Juan de Jesús, (2003). Asociación Mexicana de Escuelas y Facultades de Medicina Veterinaria y Zootecnia. Imagen Veterinaria, 3(2), 4-7. Recuperado de http://www.fmvz.unam.mx/fmvz/imavet/v3n2a03/ivv3n2a03.pdf\#page=5 
Universia Colombia. (13 de octubre, 2015). Descubre cuántas horas deberías estudiar por día. Recuperado

de http://noticias.universia.net.co/educacion/noticia/2015/10/13/1132274/descubrecuantas-horas-deberias-estudiar-dia.html

Universidad Autónoma Metropolitana, Unidad Xochimilco, México. (2013). Plan de estudios de la Licenciatura en Medicina Veterinaria y Zootecnia. Xochimilco, México: División de Ciencias Biológicas y de la Salud. Departamento de Producción Agrícola y Animal. Coordinación de Medicina Veterinaria y Zootecnia.

Zamudio, Daniel y Alvarado, Víctor. (2014). ZMVM: Hacia el colapso vial. México: El Poder del Consumidor. Recuperado de http://elpoderdelconsumidor.org/wpcontent/uploads/2014/11/ColapsoVial final.pdf

\section{Anexo 1}

\section{CUESTIONARIO SOBRE ACTIVIDADES RUTINARIAS DEL ALUMNADO}

La información proporcionada en este cuestionario es para uso estrictamente académico, con carácter anónimo y confidencial.

1. Entendiendo por rutinas, los quehaceres o actividades habituales en que ocupas la mayor parte de tu tiempo, señala a continuación:

a) La naturaleza (tipo) de cada una de las sieteprincipales rutinas a que estás habituado o acostumbrado (sólo marca con una "X");

b) la descripción (brevemente, en qué consiste) y el sitio donde la llevas a cabo;

c) el número de veces que realizas cada rutina (por día o semana); y

d) el tiempo que le dedicas en cada ocasión.

\begin{tabular}{|l|l|l|l|}
\hline $\begin{array}{l}\text { Naturaleza de la rutina. } \\
\text { (Marca con una "X") } \\
1 \ldots 7^{4}\end{array}$ & $\begin{array}{l}\text { Descripción de la rutina } \\
\text { (menciona brevemente en } \\
\text { qué consiste). }\end{array}$ & $\begin{array}{l}\text { Número de veces que } \\
\text { la realizas (especifica } \\
\text { por día o por } \\
\text { semana). }\end{array}$ & $\begin{array}{l}\text { Tiempo que } \\
\text { le dedicas a }\end{array}$ \\
cada ocasión.
\end{tabular}

\footnotetext{
${ }^{4}$ El cuadro se repite siete veces.
} 
2. ¿En una escala de 1 a 5, donde 1 es muy malo, 2 malo, 3 regular, 4 bueno, y 5 muy bueno, cómo calificas tus siguientes habilidades?:

- Lectura y comprensión...............( )

- Escritura y redacción..................( )

- Comunicación interpersonal.........( )

- Matemáticas........................... ()

- Creatividad........................... ()

- Trabajo en equipo.....................( )

- Tecnologías de la información y la comunicación ...................... ( )

3. ¿De las siguientes fuentes de información, asigna los números 1,2 y 3, a las que consideras tus principales fuentes de aprendizaje?

- Libros especializados.

- Materiales proporcionados por los docentes.

- Internet (artículos y documentos varios).............. ( )

- Revistas científicas.................................( )

- Notas de clase....................................... ()

- Apuntes de compañeros............................. ()

- Artículos científicos (biblioteca)..................... ( )

- Artículos técnicos (biblioteca)....................... ( )

- Otra (especifica)__... ( )

4. ¿En lo que va de tus estudios en la UAM Xochimilco, cuántos módulos has cursado? ¿Cuántos módulos has reprobado?____ ¿Cuál es tu promedio actual (en número)?

UAM-Xochimilco, a 29 de agosto de 2016. 